\title{
Rorschach Assessment of Two Distinctive Personality States of a Person with Dissociative Identity Disorder
}

Dissociative Identity Disorder (DID; Diagnostic and Statistical Manual of Mental Disorders, 5th ed., American Psychiatric Association, 2013) is described as a fragmentation of the identity characterized by the presence of two or more distinct identities or personality states. Each state has its own and relatively enduring pattern of perceiving, relating to, and thinking about the self and the environment. At least two of these personality states recurrently take control of the person. According to the guidelines for treating dissociative identity disorder in adults (International Society for the Study of Trauma and Dissociation, 2011), alternative identities have been defined in a number of ways. These include for example personality states, alter personality and dissociative parts of the personality. Early dissociation may represent an adaptive reaction to an inescapable threat or danger in which flight or fight is impossible (Brand, Loewenstein, \& Lanius, 2014). Serious neglect, withdrawal, and abuse from caregivers, resulting in disorganized attachment seem to predict adult dissociation (Lyons-Ruth, 2015). There exist several theories about dissociation (see e.g., Dell, 2009). In this project, we found the theory of trauma-related structural dissociation of the personality (van der Hart, Nijenhuis, \& Steele, 2006) useful. This theory views dissociation as a mechanism occurring during and after overwhelming strains to body and mind, resulting in fragmentation of the developing personality. The fragmentation functions to meet the needs of ongoing daily life, splitting off painful emotions, and bodily stored trauma memories. The personality states that function primarily in daily life are mediated by action systems such as working, taking care of children, social functioning and acts to suppress or avoid traumatic memories, often called "The going on with normal life or daily life-parts (Steele, Boon, van der Hart, 2017; Fisher, 2017). This theory uses the term "personality parts" as it views the core structure of complex dissociative disorders to involve 
unusual strong separation, including separate senses of self with their own first-person perspective (a sense of $I$, me, and mine) and amnesia between the separated parts. In the following, we will however, in agreement with the DSM-5 use the concept of personality "states" as an umbrella category including dissociative parts.

According to the structural dissociation theory, overwhelming strains to the person may cause some personality states to be fixated on biological survival, such as attachmentcry, flight, fight, and/or submission, here called trauma-related states (van der Hart et al., 2006). Due to not being processed, bodily stored memories are stuck in the past, thereby preventing the daily life state from knowing that the neglect/abuse is over. Fragmented states may contain behavior and perceptions from early childhood and adolescence, with their own sense of self and patterns of mental functioning. Additional fragmentation may occur during the lifespan to manage challenges such as puberty, sexual relations, parenthood, school/work, and new traumatization. Cognitive functions may develop continuously, whereas emotional responses and needs are suppressed, often living a hidden and lonely life on the inside of the individual. The person may develop several psychological and somatic symptoms due to the split of the different elements usually integrated in the ongoing personality development. Phobia against realizing earlier neglect and traumas may cause depression, fear of intimacy, time gaps, and confusion in daily life.

\section{The Case}

Our case involves a highly intelligent 50-year-old woman working fulltime as an engineer in a large company. She was referred to therapy to the second author after barely surviving an assault rape by several offenders and was still in the same psychotherapy, which had lasted for periods of varying lengths during 10 years. The patient met the criteria of DID according to the DSM-IV, Text Revision assessed by SCID D (American Psychiatric Association, June 2000). When she started in therapy, she was seriously depressed, performed 
severe self-harming and suicidal behaviors, and had symptoms of hearing her father's voice, shifting personality states, and posttraumatic stress disorder (PTSD; Briere, 2004).

Different states of the patient's personality took control over the body, creating almost complete amnestic barriers between them. The patient's personality states associated with daily life, here called "Ann", were striving to take care of the functioning of daily life and work. The other personality state, here called "Ben", viewed himself as a much younger male person, eager to come more forward, be acknowledged, and listened to in therapy sessions. He had a strong defense strategy with a phobic urge against physical and psychological closeness, understood as being due to traumatic experiences (the trauma-related state. In certain periods, he also reported paranoid ideas about his father and other dangerous people invading their home. Ben was also responsible for several practical functions at home, and had high skills in handicrafts (some daily-life functions). The two personality states became more acquainted with each other during therapy.

Although the two personality states had the same appearance and mostly dressed alike, they seemed different in behavior and character. Ann was a socially likable working person, whereas Ben behaved in a more frank, direct, and masculine way. He wanted acceptance as a normal person but felt rejected and not likeable to others. However, Ann described Ben as too easily irritated, not knowing how to behave, and with too direct behavior towards others. On the other side, Ben was critical towards Ann, accusing her of always trying to please other people. Two other personality states were identified during the therapy, a young boy (6-8 years) with strong attachment longings towards the therapist when he came forward in therapy sessions, and a very feminine adult personality state, who wanted to wear dresses and get compliments from men. The therapist worked with these states primarily through Ann, who had told the therapist that she totally hides her personality states from everybody even her primary family. She was afraid people would view her as "crazy" and look down upon 
her. Her friends often became confused about her behavior and asked her if she might have Tourette syndrome. She was using much energy controlling the other personality states in order to prevent them from coming forward with their own agendas. In therapy, Ann had learned to take care of the feminine personality state, allowing her to wear dresses and put on makeup when at home, whereas Ben took care of the small-boy state who liked to have toys and needed to be assured that his father was not present, and that he was now safe.

Therapy followed the guidelines developed by the International Society for the Study of Trauma and Dissociation (2011), adding therapeutic approaches such as sensorimotor psychotherapy (Ogden \& Fisher, 2015), mentalization-based therapy (Fonagy \& Target, 1997), and attachment-based therapy (Allen, 2013). Therapeutic interventions prevented several hospitalizations and possible suicidal attempts and reduced PTSD symptoms. At the time of the assessments, Ann was accepting and caring for the different personality states. She was more able to regulate her energy and her emotional activation, being aware of signals of exhaustion when in stress, taking care of herself, and listening to warning signals of suicidal impulses from different personality states. However, she was still experiencing fugue states and personality states chaotically struggling when she was tired or in conflict at work.

\section{Rorschach Research Findings in Relation to Patients with DID}

Research on individuals with DID is sparse, and personality assessment tools are still unsettled. In 1940, the clinician and researcher on hypnosis, Milton Erickson, and the psychoanalytic-oriented personality researcher David Rapaport co-wrote an article that later was published (Erickson \& Rapaport, 1980) where they examined two women with dual personalities, here called "Woman 1" and "Woman 2". The women appeared "to have organized their past experiences into two different patterns and to react alternatingly, now on the basis of one and now on the basis of the other pattern" (p. 276). The authors described the women as shifting between a primary dominant and a secondary passive personality or 
personality states. Both women were employed and viewed as competent and efficient. The second author separately tested the primary and secondary personality state of each patient in one-day intervals using the Rorschach Inkblot Method (RIM; Rorschach, 1921), as well as some additional personality and ability tests. Unfortunately, the authors reported their results without presenting any exact test scores.

The RIM data for Woman 1 indicated that the primary personality state was relatively healthy with a stable judgement and logic, and nearly no indications of anxiety and morbid images, but with considerable intellectualization. However, the secondary personality state appeared to be neurotic, characterized by an impulsive use of color, low Form plus percentage, uneven judgement and logic, and signs of anxiety, doubt, ambivalences, oppositional tendencies, and intellectualization. Both personality states of the person showed an unusually high level of interest in other humans, although for the secondary personality state this concern was loaded with insecurity and fear of others. The authors argued that Woman 1's test results were "comparable to those of two different persons with some similar character traits" (p. 274).

For Woman 2, both personality states produced constricted protocols, thereby suggesting neurotic and inhibited traits. Still, both appeared interested in other people, but they were also shy and frightened. The primary personality was the most neurotic and inhibited. Despite these differences in personality functioning, the dual states of both Woman 1 and Woman 2 seemed to be highly intelligent.

Fifty years later in their pilot RIM study with 14 patients with DID, Armstrong and Loewenstein (1990) used the Comprehensive System (CS; Exner, 1974) and an administration procedure, in which the assessor invited the test takers to let all their different personality states come together in the same testing. Thereafter, this procedure has been the norm in the RIM assessment of patients with DID. The DIDs produced a heightened number of unusual 
form quality $(\mathrm{Xu})$ and responses with multiple determinants (blends) indicating unusual, but not distorted, reality testing combined with flexibility in thinking. Moreover, the findings suggested intellectualization and obsessiveness. Lastly, Form Dimension (FD) was also elevated. At that time, researchers and clinicians viewed FD as signifying a self-reflective ability, although no strong research supports this interpretation (Meyer, Viglione, Mihura, Erard, \& Erdberg, 2011).

Scroppo, Drob, Weinberger, and Eagle (1998) revealed a high number of Human Movement (M) scores among DID patients. M is usually interpreted as indicating intellectual, imaginative, and empathic resources. Opaas and Hartmann (2013) found an average mean score of $\mathrm{M}$ responses among severely traumatized refugees. However, they viewed this as a relatively high value for traumatized individuals as reflecting, "a kind of vigilance - careful attentiveness toward other people's every move, learned through years of suppression, threat, and abuse" (p. 466).

Brand and coworkers (Brand, Armstrong, \& Loewenstein, 2006, N = 100; Brand, Armstrong, Loewenstein, \& McNary, 2009, N = 67) have shown that patients with DID can be distinguished from non-patients, patients with PTSD, depressed patients, and patients with a borderline personality disorder and psychotic disorder. The DID patients' records were brief, but still quite complex, thus including a very high level of trauma-related content (high Traumatic Content Index [TCI, Armstrong \& Loewenstein, 1990]), suggesting traumatic flooding together with an attempt to avoid being completely overwhelmed. The DID patients also exhibited a high number of cooperative movements (COP), hence suggesting an ability and interest in a collaborative engagement with others. The DID patients had a moderately high level of inaccurate perception (X-\%) and deviant cognition (WSum6) and elevation of M-, indicating a modest impairment of reality testing and cognition together with a bent to 
misperception of others. Despite appearing able to modulate affects, the DID patients seemed to avoid emotional situations and scored high on FD.

\section{Research Questions}

The main purpose of this case study was to examine how the performance-based RIM might contribute to a better understanding of the personality fragmentation in a patient with DID: (a) Would it be possible to administer the RIM to two personality states (Ann and Ben) of the patient at two separate points in time? (b) Would this procedure make it possible to explore whether Ann and Ben responded with two distinct personality states with their own sense of self and with different psychological functioning, or would the protocols reflect some kind of co-consciousness between them? (c) Would the results of the Rorschach Performance Assessment System (R-PAS; Meyer et al., 2011) shed light on how our patient organized her intrapsychic splitting, the relations to others, and the daily life function? (d) Would the selfreport results of the Inventory of Interpersonal Problems-Circumplex (IIP-64; Horowitz, Alden, Wiggens, \& Pincus, 2000) also capture Ann and Ben with two discrete personality states with a different sense of self and a distinct interpersonal style? (e) How would our RPAS findings correspond to the results of previous RIM studies on patients with DID? (f) Lastly, would the assessments be useful for understanding the costs and benefits of the patient's personality fragmentation according to the theory of trauma-related structural dissociation of the personality (van der Hart et al., 2006)?

\section{Clinical Assessment Methods}

We used only two assessment tools, the RIM, administered, coded, and interpreted with the R-PAS, and the IIP-64, since extensive testing might be exhausting. To the best of our knowledge, no studies have used the IIP in the assessment of DID patients.

The Rorschach Inkblot Method has proven helpful in verifying the personality features of patients with DID (see e.g. Armstrong \& Loewenstein, 1990; Armstrong \& Kaser- 
Boyd, 2004; Brand et al., 2006; Brand et al., 2009; Scroppo et al., 1998). The method seeks to identify troublesome emotions and thoughts, and may reveal unprocessed traumatic memories that these patients usually keep out of their consciousness. This performance-based method has demonstrated a sensitivity to less overt deficits such as reality testing, disturbed thought processes, and invasive trauma-related images that may be hard to detect via observations, interviews, and/or self-report (Meyer et al., 2011; Mihura, Meyer, Dumitrascu, \& Bombel, 2013; Nørbech, Hartmann, \& Kleiger, 2017). As for individuals with severe PTSD symptoms, RIM testing of individuals with DID seems to result in a biphasic reaction to the inkblots, in which emotional flooding and/or a loss of control alternate with emotional and cognitive constriction and/or over-control (Brand et al., 2006; Kaser-Boyd, \& Evans, 2008; Meyer et al., 2011; Opaas \& Hartmann, 2013; Viglione, Towns, \& Lindshield, 2012).

A meta-analysis has shown a high test-retest stability for RIM codes (Grønnerød, 2003). A subsequent study by Hartmann, Halvorsen, and Wang (2013) has proven a moderate to high temporal stability of RIM codes over a 9-year follow-up interval for patients with recurrent depression. To the best of our knowledge, there exist no test-retest studies of patients with DID. Thus, even though RIM codes tend to be stable across subsequent administrations, it is unknown whether test-retest variability might be greater for DID patients than for other clinical and nonclinical samples. In any case, in our view huge potential differences between the RIM data of potential separate dissociative personality states will more likely be the result of continuous structural dissociation between the separate state (van der Hart, 2006) than due to random fluctuations in test responding.

The sight of the RIM inkblots upsets some traumatized test takers. They seem to fear that the images might be harmful for them (Brand et al., 2006; Opaas \& Hartmann, 2013). They often produce brief records $(\mathrm{R}<14)$, probably in order to escape activating traumatic 
memories. Consequently, researchers and clinicians have to take care to avoid brief records, while at the same time not needlessly triggering these patients' level of anxiety.

We selected the lately established R-PAS, as it is the most evidence-based and internationally oriented approach to the RIM. The administration procedure (R-Optimized) controls for the range of responses. Studies have shown that R-Optimized procedure reduces the number of brief records (Reese, Viglione, \& Giromini, 2014; Viglione, Meyer, Jordan, Converse, Evans, MacDermont, \& Moore, 2015). Except on the number of responses, ROptimized only produces a slight impact on RIM norms (Pianowski, Meyer, \& VillemoreAmaral, 2016a; Pianowski, Meyer, \& Villemore-Amaral, 2016b). The codes and interpretations have a quite high empirical and clinical support, and a program transforms the scores into Standard Scores (SS). The system also contains a marker of malingering or negative simulation (CritCont\%). Lastly, the normative data are based on globally collected adult reference samples (Meyer, Erdberg, \& Shaffer, 2007), which compared to the traditional CS norms, make nonclinical adults appear less pathological (Meyer, \& Viglione, 2008; Viglione, \& Giromini, 2016).

The IIP-64, version C (Horowitz et al., 2000) is a self-report tool that provides a brief test of the severity of interpersonal problems. We used the Norwegian version translated and validated by Monsen, Havik, Eilertsen, and Havik (2006). This tool consists of 64 statements to evaluate how relevant they are for the person (from not relevant to highly relevant). The test builds on the suggestion that relational behavior can be described by two primary dimensions, belonging/caring (continuum cold/warm) and control/dominance (continuum subordinate/dominant). IIP-64 questions are separated into two parts: The first consists of 39 items about interpersonal inadequacies or inhibitions, starting with the phrase, "It is hard for me to...." The remaining 25 items involve excesses or compulsions starting with, "Things that you do too much." The test takers designate their degree of difficulty or distress on a 
scale from zero (not at all distressing) to four (extremely distressing). The overall score, Global, indicates the level of general interpersonal problems. Moreover, eight sub-scores for various combinations of evaluations on the main dimension signify specific problems, including Domineering, Intrusive, Overly Nurturant, Exploitable, Nonassertive, Socially inhibitive, Cold/Detached, and Vindictive (Solbakken, Hansen, Havik, \& Monsen, 2011).

\section{The Assessment Procedures}

Ann and Ben gave written informed consent to take part in the study in two discrete sessions with their therapist (the second author). The identifying features of the patient have been masked to protect her confidentiality. The patient had demonstrated a capacity to let different personality states come forward in the therapy sessions. Both Ann and Ben would also appear separately from the start in different sessions. Before the assessments, the therapist met with Ann and Ben, respectively, and brought them to the assessor (the first author). With her long-standing relationship to Ann and Ben, the therapist was able to verify the presence of each personality state. The assessor administered the R-PAS in separate, sequential settings first to Ann, and three months later to Ben. It was impossible to keep the assessor blind to the fact that the R-PAS was given to two distinct personality states of one person with DID, as she was the head of the project. However, the assessor got no information about the psychological functioning of Ann and Ben before the testing, coding, and interpretation of test results took place.

Administration, coding, and interpretation followed the R-PAS guidelines. The Oslo R-PAS training group run by two certified testers jointly coded the R-PAS responses in separate settings. A month after the R-PAS testing of Ben, the therapist individually administered the IIP-64 to Ann and Ben in two distinct settings. Thereafter an IIP researcher interpreted the IIP-64 records. The R-PAS coding group and the IIP-64 interpreter were led to believe that they scored a female and a male protocol, thus being blind to the fact that the 
protocols were produced by two dissociated states of one female patient with DID. They became highly surprised when the authors revealed this information.

\section{The R-PAS Administration}

\section{Administration Behaviors}

The assessor administered the R-PAS in Norwegian. To ensure an accurate translation of the original English administration text, we used the Norwegian transcript that the Oslo RPAS group previously had prepared by for certification of Norwegian R-PAS assessors.

As can be seen from Ann and Ben's R-PAS Responses (see Appendix A and B), Code Sequences (see Appendix C and D), and Profile Pages (see Tables 1 and 2), Ann and Ben behaved and responded very differently during the R-PAS assessments, which lasted 50 minutes for Ann and 30 minutes for Ben.

Insert Tables 1 and 2 about here

Ann had never seen the RIM images, heard about the method, or taken the test, and wondered how responses to inkblots could convey information about her and the alter personality state. Still, she was engaged in the testing, and collaborated well. Her record (see Appendix A and Table 1) yielded an average number of 25 responses. After being prompted on Card II, Ann asked, "Does that show a lack of imagination?" and "Does it have to stay like that or can I turn it around?" She then turned the card half around and gave a response. Thereafter, she gave two to four responses to each of the remaining cards, and turned a card four more times.

Ben, to the assessor's astonishment, convincingly stated that he had not seen, heard of, or taken the RIM before. He took part in the assessment in order to be nice, and with some curiosity as to what this was all about. However, he was highly suspicious, repetitively asking whether he had to say what he saw. His record (see Appendix B and Table 2) showed many places where the assessor should ideally have asked for more clarifications. Nonetheless, Ben 
was so reluctant to explain his responses that the assessor was afraid he would leave the testing if she pushed him further. Therefore, the coding of Ben's protocol was problematic. For example, we were not sure that it would be appropriate to code his responses 16 (Card VII) and 18 (Card VIII) as Vagueness (Vg) and Form Quality none (FQn), but decided to do so.

Ben produced 20 responses, which is below the average. He had to be prompted six times (on cards I, II, V, VIII, IX, and X), which is very high and not typically due to a single personality trait. Clinical experience suggests that a high Prompts (Pr) may indicate limited cognitive abilities, rigidity, depression, lack of trust, defensiveness, and/or an uncooperative, oppositional response style. After being prompted, his reaction was commonly, "It looks like something that's not what it looks like." During the Clarification, he often became irritated, almost angry, as he did not understand why he was being questioned about his responses. His reaction was typically, "Because it's there, strange that you ask about it." He refused to give any responses to Card X. Even so, the rapport between him and the assessor was good. He stated that he had enjoyed being with her, and that it would be nice if she became his therapist.

\section{Test Validity}

Ann's record exposed surprisingly few signs of serious mental disturbances, while Ben's record revealed a high number of indices of severe psychological problems. Thus, before analyzing the R-PAS results, we looked at the possibility of positive simulation by Ann and negative stimulation by Ben. Research has specified that it is difficult for patients with mental problems to portray a healthy performance on the RIM, especially in relation to concealing perceptual and cognitive disturbance (Hartmann \& Hartmann, 2014; Nørbech, Fodstad, Kuisma, Lunde, \& Hartmann, 2016). Even so, positive simulation causes constricted protocols with a higher than average score on Pure Form (F\%) and a lower than average use 
of CritCont\%, Aggressive Content (AGC), and Aggressive Movement (AGM) (Ganellen, 2008; Hartmann \& Hartmann, 2014; Nørbech et al., 2016). Cooperative Movement (COP) may also be subject to positive impression management (Meyer et al., 2011). Thus, Ann's low scores on all codes indicating perceptual and cognitive disorders (all SS < 89), were probably not caused by positive simulation. However, her higher than average F\% (SS =112) and COP (SS = 111), low AGC (SS = 86), and average CritCont\%; SS =102) may be the result of positive impression management.

Negative simulation or malingering on the RIM is associated with a low number of responses, elevation of CritCont\%, and/or extreme behavioral and emotional reactions to the inkblots (Ganellen, 2008). These features were not largely present in Ben's record. The CritCont\% (SS = 102) was average, proposing that over-dramatization was probably not the source of his high proportion of deviant scores. The number of responses $(\mathrm{R} ; \mathrm{SS}=88)$ was low, but average when adjusted for complexity. We cannot be sure that Ben's irregular response style did not reflect attempts to appear more disturbed than he actually is. However, in accordance with his explicit desire to be normal, we viewed this behavior as honest expressions of mistrust, distress, and confusion.

Lastly, although the R-PAS assessor had no information about the psychological functioning of Ann and Ben, it was impossible for her to overlook the huge differences between Ann and Ben's test behavior, and this unavoidable fact may have resulted in some unintended assessor influence on the testing.

\section{The R-PAS Results}

\section{Engagement and Cognitive Processing}

Ann's scores in this domain were primarily within the average range, hence suggesting adequate test engagement, as well as resources such as intelligence and cognitive abilities, creativity, vitality, interest for perspective taking, and the capacity to cope with discomforting 
experiences. In addition, her protocol indicated a highly intellectualized style of information processing (IntCont; SS = 134), which may function as a defense against emotional and social stress. Her scores also signified an above average ability to manage severe difficulties (MCPPD; SS = 113).

Ben's Complexity score $(\mathrm{SS}=77$ ) was very low, indicating cognitive or coping deficits or anxiety-driven conflicts, depressive withdrawal, constriction, or traumatic numbing. Complexity adjusting the results did not meaningfully change his profile. For this reason, we primarily based our interpretations on the raw score data. His score was average on F\% (SS = 104), low on Blend $(\mathrm{SS}=84)$ and Sy $(\mathrm{SS}=74)$, and high on $\mathrm{Vg} \%(\mathrm{SS}=123)$, thus indicating an average to low openness to experience, and an impressionistic and simple style of information processing.

His coping resources appeared to be average (MC; $\mathrm{SS}=99$, MC-PPD; $\mathrm{SS}=113, \mathrm{M}$; $\mathrm{SS}=109, \mathrm{FD} ; \mathrm{SS}=104, \mathrm{~W} \% ; \mathrm{SS}=97)$. As a result, his elevated $\mathrm{Pr}$ and low Complexity most likely did not reflect a low level of intelligence. Nevertheless, one of his five Ms had a distorted Form quality (FQ-; response 19, Card IX “A spy, someone who gets involved in everything"), indicating a distorted perception of others and distrustful thinking. Although Ben appeared to have an average capacity for vitality (WSumC; SS =99), the results also indicated a tendency to become flooded by distress or taken by ideas $(\mathrm{C} ; \mathrm{SS}=114)$, and to focus on uncommon or idiosyncratic details $(\mathrm{Dd} \% ; \mathrm{SS}=123)$. He also seemed to have a very strong tendency to avoid emotional situations with other people (R8910\%; SS $=58)$.

In sum, Ann is probably able to handle the-day-to-day challenges of life in a stable and predictable but greatly intellectualized way, whereas Ben seems to be distrustful toward other people, not knowing how to react adequately, which may result in ineffective day-to-day encounters.

\section{Perception and Cognition}


Ann's scores in this domain showed a total absence of signs of perceptual and cognitive deficits, suggesting accurate perception and reality testing, and as previously noted an adequate ability to view the world as most people do, and to successfully accomplish dayto-day challenges.

Ben's protocol, in contrast to Ann's, revealed a high number of deviant scores on all codes in this domain with the exception of FQu\%. Although Ben exhibits some bizarre thinking and behavior in his daily life, the R-PAS exposed additional concealed perceptual and cognitive deficits. His Ego Impairment Index, which is a wide measure of severity in mental problems, was highly raised, due to high scores on measures indicating poor reality testing, severe disruptions in thought processes, and disordered thinking. Approximately $50 \%$ of his responses were FQ-, or did not contain an object with definite form or outline.

Additionally, four of these responses had a Cognitive Score. Thus, Ben probably has severe problems in accurate perception, logical thinking, and effective reality testing. Such a peculiar and distorted perception and cognition may result in a confused understanding of self, others, and relationships. As previously mentioned, this may undermine his ability to identify the obvious cues in his surroundings, hence making it difficult for him effectively to undertake day-to-day encounters.

Ben's four Cognitive Scores were all Deviant Responses (DR), one on Level 1 and three on Level 2. DRs are illogical or irrelevant replies to the task, "What might this be? ", which are often due to not realizing that the stimuli are just inkblots and not real-world phenomena. DR2 is an example of markedly incomprehensible communication or explanation failures that are clearly off task, such as when during the clarification of V-13 Ben stated, "It just isn't what it looks like. I think it's trying to be a kind of butterfly, but it isn't one.” This is a very odd statement, reflecting a breech in logical thinking, in which Ben seemed to have lost awareness of the interpretive characteristic of the task. A later DR2 response (IX-20) repeated 
this strange statement, indicating that for Ben the inkblots seemed to be something real, and that this real thing was not what it looked like. DR2s are often indicative of experiences of psychotic-level lapses in reasoning, communication, and thought organization. The content of Ben's responses (IX-19: “A spy, someone who gets filtered in everything”, and V-12: "Shrew" with the Clarification: "A tiny little part, it is trying to hide himself but not succeeding" and his comment on X: "I could trick them or they could fool me, therefore it is better not to say anything") indicated that his distorted cognition probably has a trauma-based delusional quality, in combination with a high level of anxiety and mental pain.

\section{Stress and Distress}

Both Ann and Ben to our surprise did not seem affected by distress. With the exception of indications of a moderate amount of internal tension ( $\mathrm{m}$; SS $=113)$, their profiles did not reveal any distracting ideation that might interfere with their reasoning. There were no indications of an experience of helplessness and distress often related to external stressors. Moreover, themes of damage, sadness, and hopelessness did not appear to bother any of them more than the average person.

We had expected an elevation of the codes in this domain for both Ann and Ben, given the patient's extremely traumatic history and complaints about problems with the altering personality states. We will return to these surprising findings in the Discussion section.

\section{Self and Other Representation}

Ann's scores were mostly in the average range, therefore suggesting that her interaction with other people appeared to be accommodating. She even seemed to have the potential for mature and healthy interpersonal relationships (Mutuality of Autonomy Health [MAH, SS = 116]).

Ben's raised and average interpersonal markers (AGM; SS = 131, Space Reversal [SR; SS = 127], M-; SS = 113, and PHR/GPHR; SS = 122) all indicated a preoccupation with 
aggressive intentions or activities, doing the opposite of what is requested, and as already pointed to - a highly disturbed understanding of self and others. Furthermore, he appeared to have little interest and need for relationships with others, and expressed sparse personal needs for guidance, support, or other types of psychological care from other people (ODL, SS = 89; $\mathrm{COP}, \mathrm{SS}=88 ; \mathrm{NPH} / \mathrm{SumH}, \mathrm{SS}=87 ; \mathrm{An}, \mathrm{SS}=85)$.

Yet, on Card VI during the Response Phase, Ben uttered in a low voice, "It is so soft." When the assessor repeated this during the Clarification, Ben said tenderly, "Want to do this" (stroked the image cautiously with his hand), and gently said, "Fur." Because Ben had told his therapist that he is fearful of psychological and physical closeness, we viewed these reactions indicating unconscious longings for attachment. Ben's five Ms suggested a kind of careful attentiveness toward other people, although he attempts to avoid becoming involved with anybody.

\section{Clinically Significant Differences between the R-PAS Records of Ann and Ben}

The profiles of Ann and Ben were very different, as they had no response completely in common. Even the locations of their responses were often unalike. The foremost differences between their records appeared in the Perception and Cognition domain. All of Ann's scores suggested no signs of perceptual and cognitive deficits, whereas all but one of Ben's scores $(\mathrm{FQu} \%)$ were severely deviant, hence indicating serious perceptual and thinking difficulties. On nine of 10 codes in this domain, the variation in SS values between Ann and Ben was larger than two standard deviations $(\mathrm{SD}=20)$, and differed from $\mathrm{SS}=34(\mathrm{FQo} \%)$ to $\mathrm{SS}=57(\mathrm{SevCog})$. We found equivalently large differences in the Engagement and Cognitive Processing domain (R8910\%, Dd\%, Vg, Blend, Complexity, M/MC, W\%, SI, IntCont, and WSumC), the Self and Other Representation domain (PHR/GHR, SR, MAH, and AGM), and for the Administration Behaviors code Pr. Only in the Stress and Distress domain were the scores of Ann and Ben equivalent. 
In sum, our findings indicated that compared to Ben, Ann seemed to have acquired an ability to view the world adequately, be cooperative, and accomplish day-to-day encounters quite efficiently. When not distressed, she even showed potential for mature and healthy interpersonal relationships and above average capacity to cope with severe difficulties, although in a highly intellectualized way. Ben on the other hand, although demonstrating average coping resources and vitality, showed many signs of severe mental problems. He appeared to have serious perceptual and cognitive difficulties - probably with psychotic-level lapses in reasoning, communication, and thought organization. His understanding of self and

others seemed to be very distorted and filled with hostile ideas and intent to do the opposite of what is demanded. He also seemed to be suspicious, anxious, lonely, and afraid of becoming emotionally involved with other people, though with some concealed longings for closeness.

\section{Interpretation of the IIP-64 Profiles}

The IIP-64 revealed Ann and Ben as having two different interpersonal profiles (see Figures 1 and 2). Ann`s profile seemed to be average, whereas Ben`s was highly deviant.

Insert Figure 1 and 2 about her

Ann's highest scores (see Figure 1) were in the Nonassertive dimension, in which she scored quite distressing on four out of 13 items, e.g., "It is hard for me to feel safe when I am together with other people." She seemed to be evasive, with tendencies to avoid commitments and having problems in saying no, disagree, and uttering her own opinion. The only other elevated domain was Socially Inhibited, in which she scored quite distressing on "It is hard for me to participate in groups." All her other scores were within the normal range.

Ben's profile (see Figure 2) showed highly elevated scores on the Cold, Socially Inhibited, and Vindictive dimensions. He scored quite or extremely distressing on five out of eight items on Cold, for example extremely distressing on "It is hard for me to experience love towards another person". On Socially Inhibited, he scored quite or extremely distressing 
on four out of seven items, e.g., "It is hard for me to open up and talk about my feelings with others." On Vindictive, he had two extremely distressing scores out of eight items, e.g., "It is hard for me to trust other people." Even more remarkably, he scored not at all distressing on all the items in the Overly Nurturant dimension, which starts with Things that I do too much, e.g., "I let another person's misery too easily affect me." His other scores indicated being suspicious, detached, withdrawn, cold, angry, stubborn, and not being relationally oriented, but rather socially avoidant.

To sum up, the IIP profiles showed two quite different sets of self-other- relational styles. When Ann is in control and not activated, she is functioning quite adequately in her daily life. When Ben is taking over the control of the body, he may probably show very deviant social behavior or have a strong tendency to withdraw.

\section{Discussion}

We explored six research questions. (a) Would it be possible to administer the RIM to two personality states (Ann and Ben) of the patient at two separate points in time? (b) Would this procedure make it possible to explore whether Ann and Ben responded with two distinct personality states with their own sense of self and with different psychological functioning, or would the protocols reflect some kind of co-consciousness between them? (c) Would the results of the Rorschach Performance Assessment System (R-PAS; Meyer et al., 2011) shed light on how our patient organized her intrapsychic splitting, the relations to others, and the daily life function? (d) Would the self-report results of the Inventory of Interpersonal Problems-Circumplex (IIP-64; Horowitz, Alden, Wiggens, \& Pincus, 2000) also capture Ann and Ben with two discrete personality states with a different sense of self and a distinct interpersonal style? (e) How would our R-PAS findings correspond to the results of previous RIM studies on patients with DID? (f) Lastly, would the assessments be useful for understanding the costs and benefits of the patient's personality fragmentation according to 
the theory of Trauma-related Structural Dissociation of the Personality (van der Hart et al., 2006)?

We and will discuss the research questions in the same order: (a) It is problematic to decide if just Ann and not any of the other personality state contributed during the first administration of the R-PAS and the IIP-64, and likewise just Ben during the second administration of the tests. Since the therapist had used her established way of bringing forward Ann and Ben, she had every reason to believe she was taking Ann to the first- and Ben to the second administration of the R-PAS, and giving the IIP-64 to Ann and Ben, respectively. The RIM assessor was also convinced that she tested two quite different personality states at the first versus the second R-PAS testing. Still, we cannot completely rule out the possibility of all states more or less collaborating during the assessments. It might for example, have been the little 6-8 year old boy with strong attachment longings who had come forward and produced the puzzling responding about fur and softness to card VI.

(b and c) The results exposed two very diverse R-PAS profiles for Ann and Ben. The profile of Ann recognized a highly intellectualized style of information processing with few other indications of deviant psychological functioning and signs of potentials for mature relationships, and above average capability to manage challenges. However, Ben's profile revealed several signs of severe perceptual, cognitive and interpersonal difficulties. His distorted cognition, understanding of self and others, and relational style probably had a trauma-based delusional quality, in combination with high levels of suspicion, anxiety, loneliness, and pain. Both profiles reflected average intelligence. Highly unexpected, except for indications of a moderate amount of internal tension, all scores on the Stress and Distress codes were average for both Ann and Ben. Even CritCont\%, which is very similar to TCI (CS) and associated with trauma, abuse, and dissociative inclinations, was average. (d) The 
IIP-64 results, like the R-PAS results, showed divergent mental capacities for Ann and Ben, and further documented relational difficulties of emotional withdrawal on Ben`s behalf.

RIM scores tend to be stable across time. As there exist no test-retest data for patients with DID, we do not know if the test-retest variability for DIDs may vary more than for other clinical and nonclinical individuals. However, we find it difficult to believe that those great differences between the first and second testing as we found for the R-PAS and the IIP-64 were simply due to test-retest variability. We also find it unlikely that an individual with no knowledge of the RIM and the IIP-64 would be capable of faking these test results. It would be even less plausible to suggest that our patient had a mental breakdown during the second administration of the R-PAS and of the IIP-64. The assessors did not observe any indications of severe breaks during these assessments. We therefore believe we tested two separate personality states of a patient with DID.

Asking Ann and Ben to take the tests separately may have exaggerated the differences between their test responding. The patient may have tried to comply with what she thought we wanted her to do or by her own wish to overstate the differences between her personality states. However, as pointed out earlier, we concluded that Ann's total absence of scores indicating perceptual and cognitive deficits was probably not caused by faking good. Positive simulation is especially difficult to accomplish on the perceptual and cognitive RIM codes. On the other hand, Ben's high number of deviant scores and peculiar response style on the RPAS probably were not due to trying to appear disturbed, as that would be contrary to the patient and Ben's wish to appear unremarkable.

Both the R-PAS and the IIP-64 assessors knew that they were testing two dissociated states of a person with DID. These biases could have implicitly affected the patient's test behavior. However, the R-PAS assessor's strongest expectations were that both Ann and Ben would score high on CritCont\% and that the protocols of both personality states would show 
signs of cognitive and emotional disturbances. In addition, it was surprising for the therapist that Ben responded as deviant as he did on both tests.

(e) Compared to previous RIM research with patients with DID, our findings were very similar to the outcomes of the two case studies by Erickson and Rapaport (1940), which used a comparable administration procedure to ours. We were particularly amazed that the RPAS data for Ann and Ben, like the RIM data for the primary and secondary personality state of Woman 1, were analogous to those of two separate individuals with some similar personality traits. In addition, the findings for Ann were almost identical with the results for the primary personality state of Woman 1 , which in both cases suggested a rather healthy personality with firm cognition, nearly no indications of anxiety or morbid fantasies, but with a highly intellectualized coping style. In addition to experiencing himself more male than female, Ben compared to the secondary state of Woman 1, seemed to have serious perceptual, cognitive, and interpersonal deficiencies. Nevertheless, both Ben and the secondary state of Woman 1delivered few responses with ordinary form quality and a high number of human movement responses, and both showed signs of oppositional tendencies. As in our patient, the records of Woman 1 and Woman 2 seemed to contain few trauma-related content responses, as such issues are not reported on, except that the primary state of Woman 1 had "a not morbid fantasy life" (p. 278).

Most RIM research on individuals with DID does not test the patients in separate settings, but instead invites patients to let all their personality states join in the same assessment (see e.g., Armstrong \& Loewenstein, 1990; Brand et al., 2006; Brand et al., 2009; Scroppo et al., 1998). The administrative procedures of the traditional RIM research on patients with DID are very different from our procedure, and whereas their protocols were scored according to the CS, we used the R-PAS guidelines. Still, we found it strange that our study revealed an average amount of traumatic content (CritCont\%) for both Ann and Ben, 
which stands in contrast to these studies' high scores on TCI, which is very similar to CritCont $\%$. There may be substitute explanations for this discrepancy. The low proportion of the CritCont\% by Ann may be due to her intellectualized coping style and her possible tendency to impression management, thereby resulting in both unconscious and conscious efforts to hold back traumatic images. Likewise, Ben's resistance to give responses and to clarify his responses, may be the result of holding back responses with trauma-related contents, which for various reasons he believed were dangerous or inappropriate to expose. On the other hand, the CritCont\% is a code with a moderate evidence basis (Meyer et al., 2011). It is probable that the CritCont $\%$ often does not catch trauma-related contents since such contents may be linked to traumatic experiences in an individualized manner that the CritCont\% may not always catch.

The low CritCont\% score of Ann and Ben may however be related to the fact that our patient had been in psychotherapy for a long time, although only recently therapist and patient have been able to work on the traumas. Opaas and Hartmann (2017) in an after-therapy termination follow-up study of severely traumatized refugees showed that the mean score on CritCont $\%$ among other R-PAS codes had substantially decreased from pre-treatment to after psychotherapy termination, although the mean was still more than one SD higher than the TCI mean of the reference sample of Meyer et al. (2007).

(f) Regarding our last research question, and in agreement with the high test-retest stability for RIM codes (Grønnerød, 2003; Hartmann et al., 2013), we found it unlikely that the extreme divergence between the R-PAS profiles for Ann and Ben was caused by random variations in test responding. On the contrary, we think both the R-PAS and the IIP-64 results are in agreement with the theory of structural dissociation (Steele et al., 2017; van der Hart et al., 2006). This theory implies an enduring split in the organization of the personality with more or less separate entities with their own sense of self, perception of the world, and ways 
of organizing emotional, cognitive, and social functions. In this theoretical perspective Ann and Ben would be viewed as two discrete personality "parts" and not "states". The theory of structural dissociation views dissociation as a way of responding to ongoing overwhelming strains of a person. By splitting off painful emotions and bodily-stored trauma memories to one state of the personality, another personality state becomes more enabled to meet the needs of daily life. Ann's intellectualizing defense strategy seems to be effective, but with a high cost of energy. Even if she may function in her daily life, she suffers from loneliness, avoiding commitments, and not being able to calm down or focus on her emotions and inner life, and uttering her own opinions. Ben's state of the personality system seems to suffer from perceptional and cognitive deficits with limited access to the daily life resources of information, but also showed signs of average coping resources. The DID fragmented personality structure can be seen as both a defense strategy and a highly developed capacity for adapting to unbearable strains, in addition to a pathological pathway in the personality development that produces serious psychological pain and symptoms.

We speculated as to whether some of the divergence between the R-PAS result of our case and the RIM research data of patients with DID may be due to the use of two different administrative procedures (testing the dissociative states of patients with DID in separate settings versus inviting all the states to participate in the same testing). Because our patient appeared clearly fragmented during the therapeutic process, we thought we had the opportunity to test Ann and Ben in separate settings. By this, we somehow "accepted" the split in personality structure, and obtained a possibility to observe the more or less distinct functioning of Ann versus Ben's responding to the R-PAS and IIP-64 tasks. However, clinical experience with- and research on patients with DID have demonstrated that the personality fragmentation of DIDs may vary, hence making it difficult or impossible to test different personality states in separate settings. When researchers let all the dissociative states of a 
patient take part in the same RIM or other personality assessments, the test results may reflect a mixture of the various states' impact on the patient's way of responding to the test tasks. It would have been interesting to administer the R-PAS and IIP-64 once more to our patient using the standard DID procedure of inviting her to let all her personality states join in the assessments. Such a retest might have shown whether the record would reflect a mixture of Ann and Ben's previous contributions.

Especially our R-PAS but also the IIP-64 assessment appeared to be clinically useful in revealing underlying, covert severe mental problems affecting the entire patient, but kept away from conscious knowledge of the daily life functioning. Clinical implications would imply taking into consideration that the different personality states have their individual characteristics, relational styles, and levels of functioning. Thus, therapists must relate differently to the various personality states and be aware that the various states may trigger different countertransference reactions, while at the same time keeping in mind that there is only one person and one body. The R-PAS and IIP-assessments may help therapists to get a clearer picture of the various DID states' functioning, which may help them to better understand and relate to the patient. In addition, therapists must adapt their interventions to each personality state, taking into account that there is often a phobic barrier and a "not knowing- relationship" between the personality states. Using psychoeducation with special emphasis on explaining the theory of personality states had an important normalizing and dignifying function on our patient. The patient felt better understood and more accurately seen by her therapist and more able to acknowledge that her traumatization mostly is carried by the Ben personality state. This understanding helped her slowly to validate and integrate her trauma history. Getting patients with DID to know their different inner states, and acknowledge their importance as survival strategies, is crucial for the therapeutic healing process. 


\section{References}

Allen, J. G. (2013). Restoring metalizing in attachment relationships: treating trauma with plain old therapy. Washington, DC: American Psychiatric Publishing.

American Psychiatric Association. (2013). Diagnostic and statistical manual of mental Disorders Text Revision $\left(5^{\text {th }}\right.$ Ed.). Arlington, VA: American Psychiatric Association. American Psychiatric Association. (June 2000). Diagnostic and statistical manual of mental Disorders Text Revision (4 ${ }^{\text {th }}$ Ed.). Washington, DC: American Psychiatric Publishing.

Armstrong, J. G., \& Kaser-Boyd, N. (2004). Projective assessment of psychological trauma. In: M. Hilsenroth \& D. Segal (Eds.), Comprehensive handbook of psychological assessment, Vol. 2. Objective and projective assessment of personality and psychopathology (pp. 500-512). New York, NY: Wiley.

Armstrong, J. G., \& Loewenstein, R. J. (1990). Characteristics of patients with multiple personality and dissociative disorders on psychological testing. Journal of Nervous and Mental Disease, 178, 448-454.

Brand, B. L., Armstrong, J. G., \& Loewenstein, R. J. (2006). Psychological assessment of patients with dissociative identity disorder. Psychiatric Clinics of North America, 29, $145-168$.

Brand, B. L., Armstrong, J. G. Loewenstein, R. J., \& McNary, S. W. (2009). Personality differences on the Rorschach of dissociative identity disorder, borderline personality disorder, and psychotic inpatients. Psychological Trauma: Theory, Research, Practice, and Policy, 1, 188-205.

Brand, B. L., Loewenstein, R. J., \& Lanius, R. A. (2014). Dissociative identity disorder. In G. O. Gabbard (Ed.), Treatments of psychiatric disorders (pp. 439-458). Washington DC: American Psychiatric Publishing.

Briere, J. (2004). Psychological Assessment of Adult Posttraumatic States: Phenomenology, 
diagnosis, and measurement ( $2^{\text {nd }}$ Ed.). Washington DC: American Psychological Association.

Dell, P. F. \& O’Neil, J. A. (Eds.) (2009). Dissociation and dissociative disorders. DSM-V and beyond. New York, NY: Routledge.

Erickson, M., \& Rapaport, D. (1980). Findings on the nature of the personality structures in two different dual personalities by means of projective and psychometric tests. In E. L. Rossi (Ed.). The collected papers of Milton Erickson: Vol 3: Investigations of psychodynamic processes (pp. 271-286). New York: Irvington.

Exner, J. E. Jr. (1974). The Rorschach: A Comprehensive System: Vol. 1. New York: Wiley. Fisher, J. (2017). Healing the Fragmented Selves of Trauma Survivors. Overcoming SelfAlienation. New York: Routledge.

Fonagy, P. \& Target, M. (1997). Attachment and reflecting function: Their role in self organization. Developing Psychopathology, 9: 679-700

Ganellen, R. J. (2008). Rorschach assessment of malingering and defensive response set. In C. B. Gacono \& F. B. Evans (Eds.). The Handbook of Forensic Rorschach Assessment (pp. 89-119). New York: Routledge, Taylor \& Francis Group.

Grønnerød, C. (2003). Temporal stability in the Rorschach method: A meta-analytic review. Journal of Personality Assessment, 80, 272-293.

Hartmann, E., Halvorsen, M., \& Wang, C. E. (2013). Rorschach variables and dysfunctional attitudes as measures of depressive vulnerability: A 9-year follow-up study of individuals with different histories of major depressive episodes. Journal of Personality Assessment, 95, 26-37.

Hartmann, E., \& Hartmann, T. (2014). The impact of exposure to Internet-based information about the Rorschach and the MMPI-2 on psychiatric outpatients' ability to simulate mentally healthy test performance, Journal of Personality Assessment, 96, 432-444. 
Horowitz, L. M., Alden, L. E., Wiggins, J. S., \& Pincus, A. L. (2000). IIP-64/IIP-32

professional manual. San Antonio, TX: The Psychological Corporation.

International Society for the Study of Trauma and Dissociation. (2011). Journal of Trauma \& Dissociation, 12, 115-187.

Kaser-Boyd, N., \& Evans, F. B. (2008). Rorschach assessment of psychological trauma. In C.

B. Gacono \& F. B. Evans (Eds.). The handbook of forensic Rorschach assessment (pp. 255-277). New York, NY: Taylor \& Francis.

Lyons- Ruth, K. (2015). Dissociation and parent-infant dialogue: A longitudinal perspective from attachment research. Attachment, 9, 253-276.

Meyer, G. J., Edberg, P., \& Shaffer, T. W. (2007). Toward international normative reference data for the Comprehensive System. Journal of Personality Assessment, 89(S1), S201S216.

Meyer, G. J., \& Viglione, D. J. (2008). An introduction to Rorschach Assessment. In R. P. Archer \& S. R. Smith (Eds.), Personality Assessment, (pp. 281-336). New York, NY: Routledge.

Meyer, G. J., Viglione, D. J., Mihura, J., Erard, R. E., \& Erdberg, P. (2011). Rorschach Performance Assessment System: Administration, coding, interpretation, and technical manual. Toledo, OH: Rorschach Performance Assessment System.

Mihura, J. L., Meyer, G. J., Dumitrascu, N., \& Bombel, G. (2013). The validity of individual Rorschach variables: Systematic reviews and meta-analysis of the Comprehensive System. Psychological Bulletin, 139, 548-605. doi:10.1037/a0029406

Monsen, J. T., Havik, O. E., Eilertsen, d. E., \& Hagtvet, K. (2006). Circumplex structure and personality disorder correlates of the Interpersonal Problems Circumplex Model (IIPC). Psychological Assessment, 18, 165-173. 
Nørbech, P. B., Fodstad, L., Kuisma, Lunde, I., \& Hartmann, E. (2016). Incarcerated violent offenders' ability to avoid revealing their potential for violence on the Rorschach and the MMPI-2. Journal of Personality Assessment, 98, 419-429.

Nørbech, P. B., Hartmann, E., \& Kleiger, J. H. (2017). Using R-PAS in the assessment of possible psychosis and trauma intrusions in a psychopathic female. In J. L. Mihura \& G. J. Meyer (Eds.) Using the Rorschach Performance Assessment System (R-PAS), (pp. 226-245). New York, NY: Guildford.

Ogden, P. \& Fisher, J. (2015). Sensorimotor psychotherapy: interventions for trauma and attachment. New York, W. W. Norton.

Opaas, M. \& Hartmann, E. (2013). Rorschach assessment of traumatized refugees: An exploratory factor analysis. Journal of Personality Assessment, 95, 457-470.

Opaas, M., \& Hartmann, E. (2017), Changes in Rorschach R-PAS Results, Symptoms, and Quality of Life of Traumatized Refugees Following Psychotherapy. Oral presentation at The XXIInd International Congress of the Rorschach and Projective Methods in Paris.

Opaas, M., Hartmann, E., Wentzel-Larsen, T., \& Varvin, S. (2016). The relationship of pretreatment Rorschach factors to symptoms, quality of life, and real-life functioning in a Three-Year Follow-Up of Traumatized Refugee Patients. Journal of Personality Assessment, 98, 247-260.

Pianowski, G., Meyer, G. J., \& Villemore-Amaral, A. E. de. (2016a). Potential projective material on the Rorschach: Comparing Comprehensive System protocols to their modeled R-Optimized administration counterparts. Journal of Personality Assessment, 98, 398-407.

Pianowski, G., Meyer, G. J., \& Villemore-Amaral, A. E. de. (2016b). The impact of ROptimized administration modeling procedures on Brazilian normative reference 
values for Rorschach scores. Journal of Personality Assessment, 98, 404-418.

Reese, J. B., Viglione, D. J., \& Giromini, L. (2014). A comparison between Comprehensive System and an early version of Rorschach Assessment System administration with outpatient children and adolescents. Journal of Personality Assessment, 96, 515-522.

Rorschach, H. (1921). Psychodiagnostics. New York, NY: Grune \& Stratton.

Scroppo, J. C., Drob, S. L., Weinberger, J. L., \& Eagle, P. (1998). Identifying dissociative identity disorder: A self-report and projective study. Journal of Abnormal Psychology, 107, 272-284.

Solbakken, O. A., Hansen, R. S., Havik, O. E., \& Monsen, J. T. (2011). Assessment of affect integration: Validation of the affect consciousness construct. Journal of Personality Assessment, 93, 257-265.

Steele, K., Boon, S., \& van der Hart, O. (2017). Treating Trauma-Related Dissociation. New York: W. W. Norton \& Company.

van der Hart, O., Nijenhuis, E. R. S, \& Steele, K. (2006). The haunted self: Chronic traumatization and structural dissociation of the personality. New York: W. W. Norton.

Viglione, D. J., \& Giromini, L. (2016). The effect of using the International versus the Comprehensive System norms for children, adolescents, and adults. Journal of Personality Assessment, 98, 391-397.

Viglione, D. J., Towns, B., \& Lindshield, D. (2012). Understanding and using the Rorschach inkblot test to assess post-traumatic conditions. Psychological Injury and Law, 5, 135144.

Viglione, D. J., Meyer, G. J., Jordan, R. J., Converse, G. L., Evans, J., MacDermont, D., \& Moore, R. (2015). Developing an alternative Rorschach administration method to optimize the number of responses and enhance clinical inferences. Clinical 
Psychology and Psychotherapy, 22, 546-558. 